\title{
Retrospective analysis of autoimmune hepatitis-primary biliary cirrhosis overlap syndrome in Korea: characteristics, treatments, and outcomes
}

\author{
Yoonsang Park, Yuri Cho, Eun Ju Cho, and Yoon Jun Kim \\ Department of Internal Medicine and Liver Research institute, Seoul National University College of Medicine, Seoul, Korea
}

Background/Aims: Overlap syndrome of autoimmune hepatitis (AlH) and primary biliary cirrhosis (PBC) (AlH-PBC overlap syndrome) is a rare disease that has not been clearly characterized in Korean patients. This study investigated the clinical features of AIH-PBC overlap syndrome compared with those of AlH and PBC alone.

Methods: This retrospective cohort study included 158 consecutive patients who were diagnosed as AlH ( $n=61)$, PBC $(\mathrm{n}=81)$, or AlH-PBC overlap syndrome $(\mathrm{n}=9)$ based on the Paris and the International Autoimmune Hepatitis Group (IAIHG) criteria from 2001 to 2011 in Korea. We compared the clinical features of these three groups retrospectively, including their biochemical characteristics, treatments, responses, and clinical outcomes.

Results: The AIH-PBC overlap syndrome patients exhibited biochemical characteristics of both AIH and PBC, and showed a similar response to ursodeoxycholic acid (UDCA) monotherapy as for the PBC patients. However, the response of AlHPBC overlap syndrome patients to UDCA and steroid combination therapy was worse than the response of AlH patients to steroid-based therapy $(P=0.024)$. Liver cirrhosis developed more rapidly in AlH-PBC overlap syndrome patients than in AlH patients group $(P=0.013)$, but there was no difference between AlH-PBC overlap syndrome patients and PBC patients. The rates of developing hepatic decompensation did not differ significantly between the groups.

Conclusions: The AlH-PBC overlap syndrome patients exhibited a worse response to UDCA and steroid combination therapy and a faster cirrhotic progression compared with AlH patients. (Clin Mol Hepatol 2015;21:150-157)

Keywords: Autoimmune hepatitis; Primary biliary cirrhosis; Overlap syndrome

\section{INTRODUCTION}

Autoimmune hepatitis (AIH) and primary biliary cirrhosis (PBC) are the distinct categories of disease with different diagnosis, treatment and prognosis. However, since the end of the 1970s, a heterogeneous disease with overlapping features of both $\mathrm{AlH}$ and PBC has been suggested: PBC with high serum aminotransferase and AlH with cholestatic pattern. ${ }^{1,2}$
Several criteria for diagnosing and predicting the prognosis of overlap syndrome have been proposed. The revised and the simplified AlH criteria from the International Autoimmune Hepatitis Group (IAIHG) are one of the most commonly used methods to diagnose overlap syndrome., ${ }^{3,4}$ Patients with PBC who were also diagnosed as probable or definite AlH had a higher rate of developing liver cirrhosis and its complications. ${ }^{5-7}$ Subsequently, a modified version, which eliminated negative points for the pres-

\section{Corresponding author : Yoon Jun Kim}

Department of Internal Medicine and Liver Research Institute, Seoul National University College of Medicine, 103 Daehak-ro, Jongno-gu, Seoul 110-799, Korea

Tel: +82-2-2072-3081, Fax: +82-2-743-6701

Email:yoonjun@snu.ac.kr

\footnotetext{
AlH, autoimmune hepatitis; ALP, alkaline phosphatase; ALT, alanine transaminase; AMA, anti-mitochondrial antibody; ANA, antinuclear antibody; AST, aspartate transaminase; CO criteria, Corpechot's criteria; GGT, gamma-glutamyl transpeptidase; IAlHG, International Autoimmune Hepatitis Group; lgG, immunoglobulin G; IgM, immunoglobulin M; INR, international normalized ratio; LKM-1, liver kidney microsomal type 1 antibody; PBC, primary biliary cirrhosis; PT, prothrombin time; SMA, anti-smooth muscle antibody; UDCA, ursodeoxycholic acid; ULN, upper limit of norma
} 
Yoonsang Park, et al.

ence of serum anti-mitochondrial antibody (AMA), has been proposed. This new criteria could predict the need of steroid treatment for the patients with PBC. ${ }^{8-10}$ Another well-established method is to apply the Paris criteria on both PBC and AlH patients. The patients who were diagnosed as overlap syndrome according to the Paris criteria showed different treatment response to conventional therapy and different prognosis from the classical diseases. ${ }^{10,11}$

However, the diagnostic value of those scores alone is still on debate, and more clinical studies are needed to overcome the shortage of knowledge about overlap syndrome. IAIHG stated that the use of the revised and the simplified criteria for AlH on overlap syndrome is inadequate, because the system was established with excluding the patients who showed tendency of PBC and other non-AlH liver diseases. ${ }^{12}$ Moreover, there was a report that the Paris criteria was not able to predict the response to ursodeoxycholic acid (UDCA) treatment. ${ }^{13}$ For the validation of the diagnostic criteria and clinical characteristics of overlap syndrome, several clinical studies from Asian countries have been reported. A study of overlap syndrome in Japan was diagnosed by a combined modified version of the IAIHG and the Paris criteria and a study in China was diagnosed by only clinical impression. ${ }^{10,11}$ In a previous study in Korea, the clinical outcome of the PBC patients with high IAIHG scores was reported, ${ }^{5}$ however, the clinical characteristics of the patients with overlap syndrome, who were diagnosed according to the systemic criteria, have not been identified. Moreover, the difference between overlap syndrome and AlH has not been fully evaluated, yet.

In this retrospective cohort study, we analyzed the clinical characteristics of overlap syndrome in Korea from a single tertiary referral center, as compared to that of AIH and PBC. The clinical outcomes of overlap syndrome including baseline characteristics, responses to treatment, and progression to liver cirrhosis were analyzed, retrospectively.

\section{PATIENTS AND METHODS}

\section{Study population and data collection}

A total of 158 consecutive patients who had been diagnosed as PBC or AlH from January 2001 to December 2011 at Seoul National University Hospital were included in this study. We compared the clinical outcomes of overlap syndrome with those of AlH and those of the PBC including biochemical characteristics, treatment modalities and responses, the incidences of progression to cirrhosis, and the incidence of hepatic decompensation, retrospectively.

For the diagnosis of AlH, both the revised IAIHG criteria and the simplified IAIHG criteria were used. ${ }^{3,4}$ If a patient met any of the criteria, then the patient was determined as probable or definite AlH patient; at least 10 points for the revised criteria and 6 points for the simplified criteria before the immunosuppressive treatment.

For the diagnosis of $\mathrm{PBC}$, at least two features of $\mathrm{PBC}$ according to the Paris criteria should be satisfied as follows; (1) serum alkaline phosphatase (ALP) $>2$ times upper limit of normal (ULN) or gamma-glutamyl transpeptidase (GGT) $>5$ times ULN; (2) a positive test for AMA; and (3) a liver biopsy showing florid bile duct lesion. $^{8}$

The patients who fulfilled both of the diagnostic criteria of AlH and $\mathrm{PBC}$ were regarded to have overlap syndrome. Patients who were younger than 18 years or older than 70 years at the time of diagnosis of either diseases, a chronic carrier of hepatitis B virus or hepatitis C virus, heavy alcohol drinkers (>25 g/day of alcohol), patients with Wilson's disease, hemochromatosis, alpha-1 antitrypsin deficiency, having extrahepatic biliary disease, chronic liver disease other than PBC and $\mathrm{AlH}$, and a history of malignancy within 5 years were excluded. Patients with a history of previous UDCA or immune-suppressant therapy were also excluded.

Biochemical results were collected from medical records, including ALP, GGT, aspartate transaminase (AST), alanine transaminase (ALT), prothrombin time international normalized ratio (PT-INR), albumin, and total bilirubin. Serum immunoglobulin $\mathrm{G}(\mathrm{IgG})$ and immunoglobulin $\mathrm{M}(\mathrm{IgM})$ were collected, if they were available retrospectively. All data at the time of diagnosis of overlap syndrome, AlH or PBC were collected, and subsequently followed up. Serologic tests were also recorded, including serum AMA, antinuclear antibody (ANA), anti-smooth muscle antibody (SMA), and liver kidney microsomal type 1 antibody (LKM-1). Serum AMA titer higher than 1:20 and serum SMA titer higher than 1:80 were regarded as a positive test. For serum ANA, more than 1:40 of serum ANA was regarded as a positive test. If available, histologic results were collected as well. All the patients who were diagnosed as overlap syndrome underwent a liver biopsy. Three overlap syndrome patients with a negative serum AMA were recorded as autoimmune cholangitis, PBC, and concurrent PBC and AlH, respectively, in the biopsy results.

The treatment regimens for each patient were collected, which were either UDCA monotherapy, steroid therapy with or without 
other immunosuppressant (steroid-based therapy), or UDCA and steroid combination therapy. The regimen for the AlH patients who received UDCA in addition to steroid were regarded as steroid-based therapy because UDCA is generally not considered as a therapeutic agent for AlH. The responses to corticosteroid with or without immunosuppressant including azathioprine were evaluated according to the $2010 \mathrm{AlH}$ guideline from American Association for the Study of Liver Disease. ${ }^{14}$ Clinical remission was defined as a disappearance of symptoms, a normalization of a serum aminotransferase, serum bilirubin, and IgG levels. Incomplete response was defined when there was no progression in clinical and laboratory findings and a remission was not achieved within 3 years of treatment. If there was clinical and laboratory features deteriorated, it was regarded as a failure. Responses to UDCA treatment were evaluated according to the Corpechot's criteria (CO criteria). ${ }^{15,16}$ The treatment was regarded as effective when serum ALP $<3$ times ULN, AST $<2$ times ULN and serum bilirubin $\leq 1 \mathrm{mg} / \mathrm{dL}$ after 1 year of UDCA treatment.
The disease progression to liver cirrhosis and the development of hepatic decompensation were analyzed. Liver cirrhosis was diagnosed when a patient exhibited typical signs or symptoms of liver cirrhosis such as ascites or variceal bleeding; imaging studies showed definite features of liver cirrhosis; and the platelet count was below $100,000 / \mu \mathrm{L}$ with splenomegaly. The presence of hepatic decompensation included jaundice, variceal bleeding, ascites, hepatic encephalopathy and hepatocellular carcinoma. The date of diagnosis of liver cirrhosis was regarded as the date of the disease progression to cirrhosis. A patient was regarded as having hepatic decompensation when the patient exhibited the symptoms that are listed above, died due to the progression of liver disease, or performed liver transplantation. The relation between mortality and liver disease was determined based on the medical record.

This study was approved by the Institutional Review Board of Seoul National University Hospital.

Table 1. Baseline characteristics of the patients with AlH, PBC, and AlH-PBC overlap syndrome

\begin{tabular}{|c|c|c|c|}
\hline & $\begin{array}{c}\text { PBC } \\
(n=81)\end{array}$ & $\begin{array}{c}\text { AlH } \\
(n=68)\end{array}$ & $\begin{array}{l}\text { Overlap syndrome } \\
\qquad(n=9)\end{array}$ \\
\hline $\operatorname{Sex}(M / F)$ & $13 / 68$ & $6 / 62$ & 0/9 \\
\hline Age (years) & $53(27-69)$ & $50(20-70)$ & $49(25-69)$ \\
\hline Follow-up time (years) & $4.7(0.0-12.4)$ & $5.3(0.0-12.7)$ & $5.8(2.4-13.0)$ \\
\hline AST (IU/L) & $65(24-419)$ & $180(31-1,530)$ & $132(53-448)^{*}$ \\
\hline ALT (IU/L) & $70(11-485)$ & $172(19-3,260)$ & $116(39-319)^{*}$ \\
\hline ALP (IU/L) & $265(59-810)$ & $128(44-305)$ & $361(119-570)^{\dagger}$ \\
\hline GGT (IU/L) & $361(156-1,405)$ & $112(23-771)$ & $418(179-616)^{\dagger}$ \\
\hline PT (INR) & $0.97(0.85-1.70)$ & $1.12(0.84-3.17)$ & $0.94(0.81-1.17)^{\dagger}$ \\
\hline Albumin (g/dL) & $4.2(2.5-4.7)$ & $3.7(2.2-4.9)$ & $4.1(3.0-4.6)^{\ddagger}$ \\
\hline Bilirubin (mg/dL) & $0.9(0.4-15.4)$ & $1.6(0.4-28.4)$ & $1.1(0.4-2.7)$ \\
\hline $\lg G(\mathrm{mg} / \mathrm{dL})$ & $1636(1011-2,892)$ & $2306(1,129-6,860)$ & $2242(1,880-3,235)$ \\
\hline $\operatorname{lgM}(\mathrm{mg} / \mathrm{dL})$ & $533(255-982)$ & $187(47-941)$ & $225(146-1,344)$ \\
\hline AMA (positive/total) & $81 / 81$ (100\%) & $4 / 59(6.8 \%)$ & $6 / 9(66.7 \%)^{*, t}$ \\
\hline ANA (positive/total) & $27 / 58(46.6 \%)$ & $55 / 67(82.1 \%)$ & $9 / 9(100 \%)^{*}$ \\
\hline SMA (positive/total) & $2 / 50(4.0 \%)$ & 24/68 (35.3\%) & $1 / 9(11.1 \%)$ \\
\hline Liver cirrhosis at diagnosis (cirrhosis/total) & 19/81 (23.5\%) & $15 / 68(22.0 \%)$ & $1 / 9(11.1 \%)$ \\
\hline
\end{tabular}

Data are presented as the median (min-max) or number/total (percent).

AlH, autoimmune hepatitis; ALP, alkaline phosphatase; ALT, alanine transaminase; AMA, antimitochondrial antibody; ANA, antinuclear antibody; AST, aspartate transaminase; GGT, gamma-glutamyl transpeptidase; IgG, immunoglobulin G; IgM, immunoglobulin M; INR, international normalized ratio; PBC, primary biliary cirrhosis; PT, prothrombin time; SMA, anti-smooth muscle antibody.

${ }^{*} p<0.01$ between PBC and the overlap syndrome.

${ }^{\dagger} p<0.01$ between AlH and the overlap syndrome.

${ }^{\ddagger} p<0.05$ between $\mathrm{AlH}$ and the overlap syndrome. 


\section{Statistical analysis}

The differences in proportion were assessed with Fisher's exact test. For the continuous variables, statistical significance was calculated with Mann-Whitney's U test. Time-to-progression of liver cirrhosis and hepatic decompensation were analyzed with the Kaplan-Meier methods, and the statistical significance was proven by log rank test. Statistical figures were calculated with SPSS ver. 21.

\section{RESULTS}

\section{Characteristics of the patients}

Among 158 consecutive patients, nine patients were diagnosed as overlap syndrome. The remainder of the patients served as controls: 81 patients with PBC and 68 patients with AlH. Basic demographic information and baseline characteristics are presented in Table 1 and further detailed characteristics on the patients

Table 2. Detailed characteristics of the patients with AlH-PBC overlap syndrome

\begin{tabular}{|c|c|c|c|c|c|c|c|c|c|}
\hline Patient number & 1 & 2 & 3 & 4 & 5 & 6 & 7 & 8 & 9 \\
\hline Sex & $\mathrm{F}$ & $F$ & $F$ & $F$ & $\mathrm{~F}$ & $F$ & $F$ & F & $\mathrm{F}$ \\
\hline Age (yr) & 25 & 62 & 49 & 36 & 69 & 47 & 49 & 51 & 62 \\
\hline Follow-up (yr) & 13.0 & 7.8 & 5.6 & 4.4 & 8.0 & 2.4 & 6.4 & 5.8 & 3.3 \\
\hline Initial diagnosis & $\mathrm{AlH}$ & $\mathrm{AlH}$ & $\mathrm{AlH}$ & Both & $\mathrm{AlH}$ & PBC & $\mathrm{AlH}$ & PBC & PBC \\
\hline AST (IU/L) & 132 & 166 & 69 & 137 & 448 & 171 & 53 & 71 & 121 \\
\hline ALT (IU/L) & 221 & 251 & 93 & 193 & 319 & 116 & 39 & 111 & 71 \\
\hline ALP (IU/L) & 361 & 270 & 119 & 340 & 127 & 526 & 570 & 376 & 562 \\
\hline GGT (IU/L) & 338 & 545 & 279 & 542 & 179 & 616 & 233 & 425 & 418 \\
\hline Albumin (g/dL) & 4.1 & 4.2 & 4.1 & 4.0 & 3.0 & 4.2 & 3.7 & 4.6 & 4.3 \\
\hline PT (INR) & 0.96 & 0.92 & 0.94 & 0.84 & 1.17 & 0.81 & 0.97 & 1.01 & 0.85 \\
\hline Bilirubin (mg/dL) & 0.9 & 0.7 & 0.4 & 1.4 & 2.7 & 1.9 & 0.6 & 1.1 & 1.3 \\
\hline $\operatorname{lgG}(\mathrm{mg} / \mathrm{dL})$ & 3235 & 1880 & - & 1933 & 2775 & - & - & - & 2242 \\
\hline $\operatorname{lgM}(\mathrm{mg} / \mathrm{dL})$ & 146 & 225 & - & 203 & 1344 & - & - & - & 494 \\
\hline AMA & - & 0 & 0 & 0 & - & - & 0 & 0 & 0 \\
\hline ANA & $1: 320$ & $1: 320$ & $1: 320$ & + & $1: 320$ & $1: 320$ & 1:160 & $1: 320$ & + \\
\hline SMA & 0 & - & - & - & - & - & - & - & - \\
\hline Treatment & $\mathrm{Comb}^{\ddagger}$ & $\mathrm{Comb}^{\ddagger}$ & $U>C^{\S}$ & $U>C^{\S}$ & $\mathrm{Comb}^{\ddagger}$ & UDCA & UDCA & UDCA & UDCA \\
\hline UDCA response & N/A & N/A & N/A & $\mathrm{R}$ & N/A & N/A & $\mathrm{R}$ & $\mathrm{N}$ & $\mathrm{N}$ \\
\hline Steroid response & 1 & 1 & 1 & 1 & $\mathrm{R}$ & N/A & N/A & N/A & N/A \\
\hline Progression to liver cirrhosis & 0 & $0^{*}$ & - & - & 0 & - & 0 & $N / A^{\dagger}$ & - \\
\hline \multicolumn{10}{|l|}{ Histological findings } \\
\hline Interface hepatitis & 0 & 0 & 0 & 0 & 0 & 0 & 0 & 0 & - \\
\hline Lymphocytic infiltration & N/A & 0 & 0 & N/A & 0 & 0 & O & 0 & 0 \\
\hline Rossetting & - & - & - & - & - & - & - & - & - \\
\hline Bile duct lesion & 0 & - & - & 0 & 0 & 0 & - & 0 & 0 \\
\hline
\end{tabular}

A positive test for AMA means at least 1:20; A positive test for ANA means at least 1:40; A positive test for SMA means at least 1:80.

AlH, autoimmune hepatitis; ALP, alkaline phosphatase; ALT, alanine transaminase; AMA, antimitochondrial antibody; ANA, antinuclear antibody; AST, aspartate transaminase; F, female; GGT, gamma-glutamyl transpeptidase; IgG, immunoglobulin G; IgM, immunoglobulin M; INR, international normalized ratio; PBC, primary biliary cirrhosis; PT, prothrombin time; SMA, anti-smooth muscle antibody; UDCA, ursodeoxycholic acid; N/A, not available; R, remission or response; I, incomplete response; $\mathrm{N}$, no response.

"This patient developed hepatic decompensation.

${ }^{\dagger}$ This patient had cirrhotic liver at the time of diagnosis.

${ }^{\ddagger}$ The combination therapy from the beginning of the treatment.

${ }^{\S}$ Corticosteroid was added after at least a year of UDCA monotherapy. 
with overlap syndrome are presented in Table 2.

One patient with overlap syndrome was initially diagnosed with having both PBC and AIH simultaneously. Three patients were initially diagnosed with having PBC, and $4.7 \%$ of the PBC patients were having overlap syndrome. The other five patients initially had $\mathrm{AlH}$, and the overlap syndrome patients were accounted for 7.3\% among all the AlH patients. The median follow-up period was 4.7 years (range, 0.0-12.4 years) and 5.3 years (range, 0.0 12.7 years) for the $\mathrm{PBC}$ and the AlH patients, and 5.8 years (range, 2.4-13.0 years) for the overlap syndrome patients, respectively. The median follow-up times were not significantly different between the groups ( $P=0.352$ for overlap syndrome vs. PBC; $P=0.321$ for overlap syndrome vs. AlH, respectively). The median ages were 53, 50 and 49 years for the $\mathrm{PBC}$, the AlH and the overlap syndrome patients, respectively, which were not significantly different between each of groups ( $P=0.516$ for overlap syndrome vs. PBC; $P=0.932$ for overlap syndrome vs. AlH, respectively).

Baseline serum ALT and AST levels of the patients with overlap syndrome were significantly higher than that of the patients with PBC ( $P=0.006$ and $P=0.009$, respectively) but not different from those of the patients with $\mathrm{AIH}(P=0.174$ and $P=0.375$, respectively). On the other hand, the baseline serum ALP and GGT levels in the patients with overlap syndrome were significantly higher than those in the patients with AlH (both $P<0.001$ ), while similar to those in the patients with $\mathrm{PBC}(P=0.237$ and $P=0.794$ respectively). Serum IgG and IgM levels were not significantly different between the groups. In addition, the patients with overlap syndrome had a higher rate of AMA positivity than the patients with $\mathrm{AlH}$ $(P<0.001)$ and had a lower rate than the patients with PBC $(P=0.001)$. The seropositive rate of ANA in the patients with overlap syndrome were significantly higher than those of the patients with PBC ( $P=0.006)$, but was not meaningfully different from those of the patients with $\mathrm{AlH}(P=0.437)$. None of the patients had a positive test for LKM-1.

\section{Treatment and response}

All the patients included in this study were treated with at least one of the following methods: UDCA monotherapy, steroid-based therapy, and UDCA plus steroid combination therapy. The proportions of the patients who were treated with each therapy are presented in Table 3.

All the patients with PBC $(n=81)$ were treated with UDCA monotherapy. Among those patients, the treatment responses were identifiable for 65 patients. The response rate of UDCA monotherapy in the PBC group was 72.3\% (47/65) according to the CO criteria. The results of corticosteroid therapy with or without UDCA were available for $45 \mathrm{AlH}$ patients. The remission rate of steroid-based therapy was $75.6 \%$ (34/45). For comparison, the remission rete according to the previous version of the AlH guideline was evaluated, ${ }^{17}$ which showed similar rate of remission (39/49, 79.6\%).

For the patients with overlap syndrome, treatment options were diverse. The treatment courses for each of patients are illustrated in Figure 1. Six patients were initially treated with UDCA monotherapy for more than 1 year, and 50\% (2/4) of patients showed treatment responses according to the $\mathrm{CO}$ criteria. The result was comparable with that of the patients with PBC $(P=0.574)$. Two patients received additional steroid-based therapy because of an incomplete normalization of liver enzyme, and five patients eventually received the combination therapy. One out of five patients (20\%) achieved a clinical remission, showing poorer response rate than the outcome of the steroid-based therapy for the patients with AIH ( $P=0.024)$.

\section{Outcomes and prognoses}

During the median follow-up period of 60 months (range, $0.2-$ 155.5 months), patients were followed-up for the development of liver cirrhosis and hepatic decompensation. Among the patients

Table 3. Numbers of patients who achieved response or remission with each therapy

\begin{tabular}{lccc} 
& \multicolumn{1}{c}{ UDCA $^{*}$} & Steroid $^{\dagger}$ & Combination $^{\dagger}$ \\
\hline PBC & $47 / 65(72.3 \%)$ & - & $3 / 3(100 \%)$ \\
AlH & $6 / 8(75.0 \%)$ & $34 / 45(75.6 \%)^{\ddagger}$ & - \\
Overlap syndrome & $2 / 4(50.0 \%)$ & - & $1 / 5(20.0 \%)^{\ddagger}$ \\
\hline
\end{tabular}

Data are presented as responders/total (percent)

AlH, autoimmune hepatitis; PBC, primary biliary cirrhosis; UDCA, ursodeoxycholic acid

*Response/total, the response was defined by Corpechot's criteria.

${ }^{\dagger}$ Remission/total, the remission was defined by AlH guideline.

${ }^{\ddagger}$ Significantly different, $P=0.024$. 
with initially non-cirrhotic liver, $24.1 \%$ (15/62) of the PBC patients and $13.2 \%(7 / 53)$ of the AlH patients progressed to liver cirrhosis during the follow-up period, respectively. Half of the overlap syn-

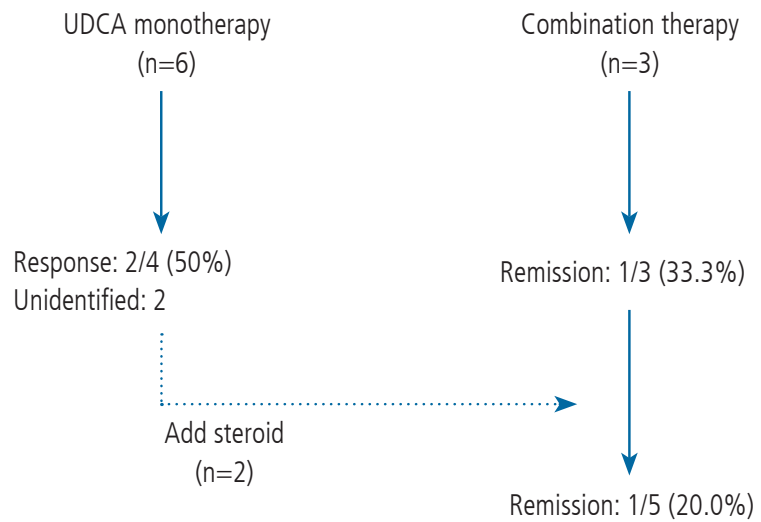

Figure 1. Treatment progress of patients with AlH-PBC overlap syndrome. UDCA, ursodeoxycholic acid; AlH, autoimmune hepatitis; PBC primary biliary cirrhosis.

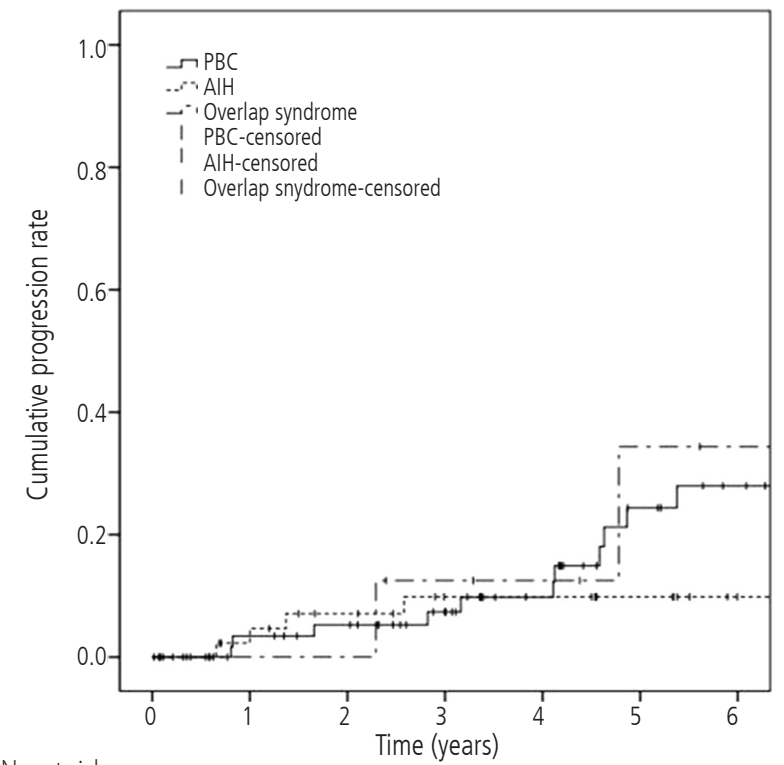

No. at risk

$\begin{array}{lccccccc}\text { PBC } & 62 & 56 & 52 & 42 & 35 & 23 & 18 \\ \text { AlH } & 53 & 40 & 36 & 31 & 27 & 24 & 18 \\ \text { Overlap } & 8 & 8 & 8 & 6 & 5 & 3 & 2\end{array}$

Figure 2. Time-to-progression curves of liver cirrhosis for patients with AlH-PBC overlap syndrome (dash-dotted line), PBC (solid line), and AlH (dashed line). The 1-year and 5-year progression rate were 3.4\% and 24.4\%, respectively, for the $\mathrm{PBC}$ patients, $4.7 \%$ and $9.8 \%$ for the AlH patients, and $0.0 \%$ and $34.4 \%$ for the AlH-PBC overlap syndrome patients. Liver cirrhosis developed significantly faster in patients with AlH-PBC overlap syndrome than in the AlH patients $(P=0.013)$. The progression rate of the AlH-PBC overlap syndrome patients did not differ significantly from that of the PBC patients $(P=0.163)$. AlH, autoimmune hepatitis; $\mathrm{PBC}$, primary biliary cirrhosis. drome patients (4/8) eventually developed liver cirrhosis. The overlap syndrome patients had a significantly shorter time-to-progression of liver cirrhosis (1-year and 5-year progression rate, $0.0 \%$ and $34.4 \%$, respectively) than that of the patients with $\mathrm{AlH}$ (1-year and 5 -year progression rate, $4.7 \%$ and $9.8 \%$, respectively, $P=0.013$ ). However, there was no difference between the patients with overlap syndrome and the patients with PBC (1-year and 5-year progression rate, $3.4 \%$ and $24.4 \%$, respectively, $P=0.163$ ) (Fig. 2).

During the follow-up period, the development of hepatic decompensation was also evaluated, including the presentation of symptoms, the incidences of liver-related mortality and liver transplantation. Among all the patients, 13.6\% (11/81) of the patients with PBC and $8.8 \%(6 / 68)$ of the patients with AlH developed hepatic decompensation during the follow-up period. For the patients with overlap syndrome, one out of the nine patients manifested the symptom of hepatic decompensation. Time-toprogression of hepatic decompensation were analyzed for each group with the Kaplan-Meier method (Fig. 3). The 1-year and 5 -year cumulative progression rates were $2.7 \%$ and $11.4 \%$ for the

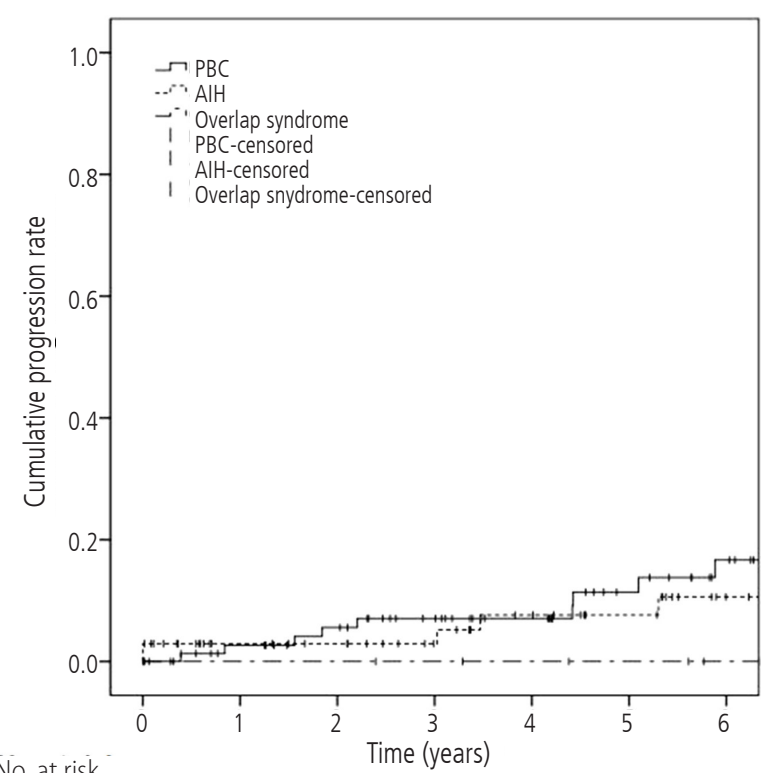

No. at risk

$\begin{array}{lccccccc}\text { PBC } & 81 & 71 & 65 & 56 & 48 & 37 & 29 \\ \text { AlH } & 68 & 53 & 50 & 48 & 37 & 32 & 22 \\ \text { Overlap } & 9 & 9 & 9 & 8 & 7 & 6 & 4\end{array}$

Figure 3. Time-to-progression curves of hepatic decompensation for patients with AlH-PBC overlap syndrome (dash-dotted line), PBC (solid line), and AlH (dashed line). The progression curve for AlH-PBC patients (1-year and 5-year progression rates both 0.0\%) did not differ from that of PBC patients (1-year and 5-year progression rates of $2.7 \%$ and $11.4 \%$, respectively; $P=0.740$ ) or $\mathrm{AlH}$ patients (1-year and 5-year progression rates of $2.9 \%$ and $7.6 \%$, respectively; $P=0.996)$. AlH, autoimmune hepatitis; PBC, primary biliary cirrhosis. 
PBC patients; $2.9 \%$ and $7.6 \%$ for the AlH patients; and both $0.0 \%$ for the overlap syndrome patients, respectively. There was no difference between each groups (overlap syndrome vs. PBC, $P=0.740 ;$ overlap syndrome vs. AlH, $P=0.996)$.

\section{DISCUSSION}

In this retrospective cohort study, the clinical outcomes and prognoses of the patients who were systematically diagnosed as overlap syndrome were evaluated as compared to those of the patients who were diagnosed as either AIH or PBC. The patients with overlap syndrome exhibited a distinct biochemical profile; baseline serum aminotransferases level in the patients with overlap syndrome were similar to that in the patients with AlH, while baseline serum ALP and GGT levels of the patients with overlap syndrome were similar to those in the patients with PBC. These characteristic profiles in laboratory test also have been observed in previous report from Japan, China, Europe and Korea. ${ }^{7,10,11,18}$ In serologic test, the difference in the patterns of serum AMA titer was consistent with previous studies, as well. ${ }^{19}$ This result represents the combined nature of overlap syndrome, not merely PBC with high serum aminotransferase or AlH with a cholestatic pattern of laboratory test.

The prevalence of overlap syndrome in this study was $4.7 \%$ among the patients with PBC and $7.3 \%$ among the patients with AlH, which has been previously reported as ranged from $3.1 \%$ to $25 \%$ in other studies. ${ }^{10,19}$ Even though the prevalence itself was not significantly different from those in the previous studies, the actual prevalence of overlap syndrome was seemed to be higher than the calculated one in this study: the IAIHG scores were underestimated in the patients with PBC because of the lack of histologic and serological results. Because of the harmless nature of UDCA, $75.3 \%$ of the patients with PBC first treated with UDCA monotherapy and then they were diagnosed based on their responses without histological diagnosis, unless the liver enzymes increased above the ULN again. Moreover, serum IgG levels were also absent in $83 \%$ of the patients with PBC. Because those results were crucial to determine the scores, the actual prevalence of overlap syndrome might be higher than the observed prevalence in this study. Similarly, among the patients with $\mathrm{AlH}$, serum AMA was not available in $13.2 \%$ of the patients, and histologic result was not available in $25 \%$ of the patients, which are the important variables in the Paris criteria. Therefore, the actual prevalence of overlap syndrome among the patients with AlH, as well, might be higher than the calculated one in this study.

The response rate to UDCA monotherapy for the patients with overlap syndrome was similar to the previous researches, ${ }^{9,10,20}$ which was lower than that for the patients with PBC. However, the difference between the groups was not statistically significant. This difference might arise from a small number of the patients with overlap syndrome included in this study. Therefore, further studies are needed to figure out the differences in clinical responses to UDCA monotherapy between the two groups.

In this study, 29 out of 45 AlH patients, whose results for steroid therapy were available, received UDCA in addition to corticosteroid. Those 29 patients were considered to be identical as the patients who were treated with steroid-based therapy and the response rate to the steroid therapy for the AlH patients was comparable to the result of previous study $(85.7 \%, 221 / 258){ }^{21}$ In our study, none of the patients with overlap syndrome received steroid-based therapy, which was previously reported to be ineffective. $^{22}$

At the end of the study, the clinical outcomes of overlap syndrome including the progression to liver cirrhosis and the presentation of hepatic decompensation were assessed. Our study suggested that the overlap syndrome patients had worse prognosis than that of the AlH patients. The patients with overlap syndrome developed liver cirrhosis with higher rate than the patients with $\mathrm{AlH}$, and the result coincided with the response to the treatment. Among all the patients who were treated with corticosteroid, the remission had a negative correlation with the cirrhotic progression rate $(P=0.019)$, which suggested a certain prognostic power of the remission criteria according to $\mathrm{AlH}$ guideline.

In this retrospective cohort study, we reported the characteristics of the overlap syndrome patients in compared to both $\mathrm{AlH}$ and PBC at the first time in Korea. The patients with overlap syndrome exhibited the clinical features of both PBC and AlH in biochemical characteristics. However, the overlap syndrome patients showed a lower rate of remission to UDCA and steroid combination therapy and significantly shorter time-to-progression of liver disease than that of the AlH patient. This study suggests a deviation between overlap syndrome and AlH in the response to the treatment and their prognosis, while the difference between overlap syndrome and PBC was not clear. Further large-volume prospective studies are warranted to clearly differentiate overlap syndrome from AlH and PBC. 


\section{Conflicts of Interest}

There is no conflict of interest or financial support to be declared.

\section{REFERENCES}

1. Geubel AP, Baggenstoss AH, Summerskill WH. Responses to treatment can differentiate chronic active liver disease with cholangitic features from the primary biliary cirrhosis syndrome. Gastroenterology 1976;71:444-449.

2. Kenny RP, Czaja AJ, Ludwig J. Frequency and significance of antimitochondrial antibodies in severe chronic active hepatitis. Digestive diseases and sciences 1986;31:705-711.

3. Alvarez F, Berg PA, Bianchi FB, Bianchi L, Burroughs AK, Cancado EL, et al. International Autoimmune Hepatitis Group Report: review of criteria for diagnosis of autoimmune hepatitis. J Hepatol 1999;31:929-938.

4. Hennes EM, Zeniya M, Czaja A, Parés A, Dalekos GN, Krawitt EL, et al. Simplified criteria for the diagnosis of autoimmune hepatitis. Hepatology 2008;48:169-176.

5. Jung HE, Jang JY, Jeong SW, Kim JN, Jang HY, Cho YJ, et al. Prognostic indicators in primary biliary cirrhosis: significance of revised IAHG (International Autoimmune Hepatitis Group) score. Clin Mol Hepatol 2012;18:375-382.

6. Neuhauser M, Bjornsson E, Treeprasertsuk S, Enders F, Silveira M, Talwalkar J, et al. Autoimmune hepatitis-PBC overlap syndrome: a simplified scoring system may assist in the diagnosis. Am J Gastroenterol 2010;105:345-353.

7. Kim MS, Kim YS, Kim SG, Byun JM, Yoon LY, Han DH, et al. The Clinical significance of simplified scoring criteria as a diagnostic tool for overlap syndrome in Korea. Korean Journal of Medicine 2013;84:211-220.

8. Chazouilleres 0 , Wendum D, Serfaty L, Montembault S, Rosmorduc O, Poupon R. Primary biliary cirrhosis-autoimmune hepatitis overlap syndrome: clinical features and response to therapy. Hepatology 1998;28:296-301.

9. Chazouilleres O, Wendum D, Serfaty L, Rosmorduc O, Poupon R. Long term outcome and response to therapy of primary biliary cirrhosis-autoimmune hepatitis overlap syndrome. J Hepatol 2006;44:400-406.

10. Tanaka A, Harada K, Ebinuma H, Komori A, Yokokawa J, Yoshizawa $K$, et al. Primary biliary cirrhosis - Autoimmune hepatitis overlap syndrome: A rationale for corticosteroids use based on a nationwide retrospective study in Japan. Hepatol Res 2011;41:877-886.

11. Wang Q, Selmi C, Zhou X, Qiu D, Li Z, Miao Q, et al. Epigenetic considerations and the clinical reevaluation of the overlap syndrome between primary biliary cirrhosis and autoimmune hepatitis. Journal of autoimmunity 2012;41:140-145.

12. Boberg KM, Chapman RW, Hirschfield GM, Lohse AW, Manns MP, Schrumpf E. Overlap syndromes: the International Autoimmune Hepatitis Group (IAIHG) position statement on a controversial issue. J Hepatol 2011;54:374-385.

13. Joshi S, Cauch-Dudek K, Wanless IR, Lindor KD, Jorgensen R, Batts $K$, et al. Primary biliary cirrhosis with additional features of autoimmune hepatitis: response to therapy with ursodeoxycholic acid. Hepatology 2002;35:409-413.

14. Manns MP, Czaja AJ, Gorham JD, Krawitt EL, Mieli-Vergani G, Vergani $D$, et al. Diagnosis and management of autoimmune hepatitis. Hepatology 2010;51:2193-2213.

15. Corpechot C, Abenavoli L, Rabahi N, Chrétien Y, Andréani T, Johanet $C$, et al. Biochemical response to ursodeoxycholic acid and long-term prognosis in primary biliary cirrhosis. Hepatology 2008;48:871-877.

16. European Association for the study of the Liver. EASL Clinical Practice Guidelines: management of cholestatic liver diseases. J Hepatol 2009;51:237-267.

17. Czaja AJ, Freese DK. Diagnosis and treatment of autoimmune hepatitis. Hepatology 2002;36:479-497.

18. Kuiper EM, Zondervan PE, van Buuren HR. Paris criteria are effective in diagnosis of primary biliary cirrhosis and autoimmune hepatitis overlap syndrome. Clin Gastroenterol Hepatol 2010;8:530-534.

19. Zhao P, Han Y. Low incidence of positive smooth muscle antibody and high incidence of isolated IgM elevation in Chinese patients with autoimmune hepatitis and primary biliary cirrhosis overlap syndrome: a retrospective study. BMC Gastroenterol 2012;12:1-4.

20. Silveira MG, Talwalkar JA, Angulo P, Lindor KD. Overlap of autoimmune hepatitis and primary biliary cirrhosis: long-term outcomes. The American journal of gastroenterology 2007;102:1244-1250.

21. Kim BH, Kim YJ, Jeong SH, Tak WY, Ahn SH, Lee YJ, et al. Clinical features of autoimmune hepatitis and comparison of two diagnostic criteria in Korea: A nationwide, multicenter study. Journal of gastroenterology and hepatology 2013;28:128-134.

22. Yokokawa J, Saito H, Kanno Y, Honma F, Monoe K, Sakamoto N, et al. Overlap of primary biliary cirrhosis and autoimmune hepatitis: Characteristics, therapy, and long term outcomes. Journal of gastroenterology and hepatology 2010;25:376-382. 